MS15-P18 Application of $\delta$-recycling to multidomain tts-microdiffraction: The ilerdite case

Jordi Rius $^{1}$, Carlos Frontera ${ }^{1}$, Oriol Vallcorba ${ }^{2}$

1. Institut de Ciència de Materials de Barcelona (CSIC), Campus de la UAB, 08193 Bellaterra, Catalonia, Spain

2. Alba Synchrotron, Barcelona, Spain

email: jordi.rius@icmab.es

In previous tts (=through-the-substrate) synchrotron microdiffraction experiments, a new aluminosilicate (code name: ilerdite) was detected in petrological polished thin sections from Tartareu (Lleida, Spain) [1]. The corresponding 2D patterns showed diffraction spots from multiple grains of ilerdite. The powder pattern was approximated by circularly integrating the 2D frames. The resulting metric of ilerdite was: Monoclinic with unit cell $a=6.398(1), b=6.904(1), c=9.427 \AA, \beta=96.42(1)^{\circ}$, $V=414 \AA^{3},\left(\mathrm{Ca}_{7} \mathrm{Mg}_{2} \mathrm{~K}_{3}\right)\left(\mathrm{Al}_{2} \mathrm{Si}_{6} \mathrm{O}_{16}\right) \cdot 5 \mathrm{H}_{2} \mathrm{O}, \quad Z=1$. Application of Patterson-function direct methods (PFDM) [2] to cluster intensities derived from whole-pattern matching gave the arrangement of the metal atoms. Due to the limited resolution of the 'powder' pattern (no reliable information fromt the high angle region) $\mathrm{O}$ atoms could not be located, so that a second tts-microdiffraction experiment at BL04 of ALBA synchrotron was performed. Details on the experimental set-up and the tts data collection mode can be found in [3]. In this experiment several microvolumes of ilerdite in different regions of the polished thin section were measured. The 2D diffraction patterns of three microvolumes were selected and processed. From this information, intensity data of six oriented ilerdite microcrystals were recovered (each microvolume contained more than one microcrystal). After multicrystal-merging, 200 single-crystal-like intensities were obtained. Application of PFDM ( $\delta$ recycling algorithm) in $P 2 / \mathrm{m}$ gave the model of the average structure which was confirmed by the subsequent LS refinement. Financial support of projects MAT2012-35247 and MAT2015-67593-P of MINECO is aknowledged. [1] Rius, J., Labrador, A., Crespi, A., Frontera, C., Vallcorba, O., Melgarejo, J.C. (2011) J. Synchrotron Rad. 18, 891-898. [2] Rius, J. (2014) IUCrJ, 1, 291-304. [3] Rius, J., Vallcorba, O., Frontera, C., Peral, I., Crespi, A., Miravitlles, C. (2015) IUCrJ, 2, 452-463.

Keywords: tts-microdiffraction, multidomain, ilerdite, structure solution, $\delta$ recycling, polished thin sections

\section{MS15-P19 HT phase transition in olivenite}

Serena C. Tarantino ${ }^{1}$, Michele Zema ${ }^{1}$, Athos M. Callegari ${ }^{1}$, Massimo Boiocchi ${ }^{2}$, Michael A. Carpenter ${ }^{3}$

1. Dipartimento di Scienze della Terra e dell'Ambiente, Università di Pavia, Italy

2. Centro Grandi Strumenti, Università di Pavia, Italy

3. Department of Earth Sciences, University of Cambridge, UK

email: serenachiara.tarantino@unipv.it

Olivenite group comprises several arsenates and phosphates minerals, such as eveite, olivenite, zincolivenite, libethenite and zincolibethenite. The members of this group crystallize in the orthorhombic Pnnm space group with the exception of olivenite, which crystallizes in the monoclinic $P 2 / n$ space group. The monoclinic symmetry of olivenite has been ascribed to a cooperative Jahn-Teller effect involving $\mathrm{Cu}^{2+}$ in an octahedral ligand field, whereby electron-phonon coupling lowers the symmetry of the structure.

In this work, a natural olivenite single crystal was submitted to in situ HT single-crystal X-ray diffraction from room temperature to $500^{\circ} \mathrm{C}$. Olivenite undergoes a structural phase transition from $P 2 / n$ to Pnnm at ca. $200^{\circ} \mathrm{C}$, and eventually becomes isostructural with the other members of the olivenite mineral group.

Instability of the electronic structure is the driving mechanism for a Jahn-Teller transition, but change in the structural state appears overtly as changes in lattice parameters, which have been analysed in terms of spontaneous strain. Moreover, the order parameter has been extracted from fractional coordinates by symmetry mode decomposition performed using the AMPLIMODES tool of the Bilbao Crystallographic Server.

There is a primary distortion (irrep GM4+), which yields the observed symmetry break between the two phases, and the full symmetric distortion mode (irrep GM1+). The amplitudes of the primary distortion is significantly large $(0.8706 \AA)$. It follows the typical law of an order parameter of a continuous phase transition $Q$ $\propto(T-T)^{0.25}$, thus indicating that the transition conforms to Landau tricritical behavior. The spontaneous strain arising in the monoclinic phase is only non symmetry breaking as the shear strain associated to the primary distortion mode GM4+ is negligible. Volume strain at room temperature is large and negative, as volume expansion with temperature is larger in the monoclinic than in the orthorhombic phase. Observed strain relationships are consistent with pseudoproper ferroelastic behaviour $V_{\mathrm{s}} \propto \mathrm{Amp} \mathrm{GM} 4+^{2}$.

Distortion of $\mathrm{Cu}$ polyhedra is quite high in the olivenite monoclinic phase at RT and goes towards a relative regularization with increasing $T$ until the phase transition occurs. At temperatures around $500^{\circ} \mathrm{C}$, the structure collapses due to dehydration and transform into the anhydrous form.

Keywords: olivenite, high temperature, single-crystal X-ray diffraction 